\title{
Testing of UBAC(2) Class of Life Distributions based on TTT - Transform
}

\author{
S.E. Abu-Youssef \\ Department of Mathematics, Faculty of Science, \\ Al- Azhar University, Cairo, Egypt
}

\author{
A.A. El-Toony \\ Department of Mathematics, Faculty of Science, \\ Helwan University, Cairo, Egypt
}

\begin{abstract}
In this paper, a new test statistic for testing exponentiality against used better than aged in increasing concave ordering $\operatorname{UBAC}(2)$ is constructed based on total time on test (TTT)Transform. Critical values are tabulated for sample size $\mathrm{n}=$ 10(5)100. The power of the test is estimated for some commonly used distributions in reliability. Finally, medical applications for real data are proposed to illustrate the theoretical results.
\end{abstract}

\section{Keywords}

UBAC(2) classes of life distributions; Survival function; Exponentiality; Total time on test (TTT)-transform; Monte Carlo method.

\section{INTRODUCTION}

Nonparametric test for testing exponentiality versus some classes of life distributions has received a good deal of attention. For testing against $\operatorname{UBAC}(2)$, we refer to IFR, DMRL, UBA, UBAE, DVRL see Bryson and Siddiqui [1], Barlow and Proschan [2], Abu-Yossef [3] and Ahmad [4].

The scaled TTT-transform and the TTT-plot were introduced by Barlow and Campo [5]. These concepts have proved to be very useful in the statistical analysis of life data.

Applications, properties and interpretations of the TTTtransform order in the statistical theory of reliability and economic can be found in Klefsjo[6,7,8] Bergman and Klefsjo [9], Pham and Turkkan [10], Bartoszewicz [11], Bartoszewicz [12], Haupt and Schabe [13], Kochar et al. [14], Li and Zou [15], Ahmed et al. [16], Li and Shaked [17], Al-Nachawati [18], Nanda and Shaked [19], Abu-Youssef et al [20], M. M. Mohie El-Din et al [21].

Let $X$ denote the life time of a specific item with distribution function $F(x)=P(X \leq t)$, survival function $\bar{F}=1-F$. and has finite mean $\mu$ and variance $\sigma^{2}$

Definition(1.1): The distribution function $F$ is said to be $\mathrm{UBAC}(2)$ if

$\int_{t}^{\infty} \bar{F}(s) d s-\int_{x+t}^{\infty} \bar{F}(s) d s \geq\left(1-e^{-x}\right) \bar{F}(t)$.

which can be written as :

$v(t)-v(x+t) \geq\left(1-e^{-x}\right) \bar{F}(t)$.

See Ali [22].

Our proposed class UBAC(2) includes many classes of life distributions. Willmot and Cai [23] showed that the UBA (used better than aged) class includes the DMRL (decreasing mean residual life) class. While Di Crescenzo [24] has shown that UBAE (used better than aged in expectation) class is contained in the HUBAE (harmonic used better than aged in expectation) class. And from Mohi el Din et al [25]. So we have:-

$$
I F R \Rightarrow U B A \Rightarrow U B A C(2)
$$

Thus we have

$$
\mathrm{IFRIFR} \subset D M R L \subset U B A \subset U B A C(2)
$$

$H U B A E \subset U B A E$

For definition and properties of these classes, you can see Deshpande et al. [26] and Deshpande and Purohit [27].

The main theme of this paper is dealing with the problem of testing $H_{0}: \bar{F}$ is exponential against $H_{1}: \bar{F}$ is $\operatorname{UBAC}(2)$ and isn't exponential based on TTT Transform.

In sec (2), the concept of total time on test transform is given. In sec (3), we derive the empirical test statistic for the UBAC (2) class of life distribution based on TTT-transform. The critical values via monte carlo method and the power of the test are tabulated in sec (4). Finally, applications using real data are also presented in sec (5).

\section{THE CONCEPT OF TORAL TIME ON TEST TRANSFORM (TTT- TRANSFORM)}

We present the following definitions of Barlow and Campo [5].

Definition (2.1): The total time on test transform (TTTTransform) of $\mathrm{F}$ is defined as

$$
H_{F}^{-1}(t)=\int_{0}^{F^{-1}(t)} \bar{F}(s) d s .
$$

where $F^{-1}(t)=\inf \{x: F(x) \geq t\}$ and $0 \leq t \leq 1$.

This transform was first studied by Barlow and Doksum [28] and Barlow et al [29]. Since the mean of $F$ is given by

$$
\mu=H_{F}^{-1}(1)=\int_{0}^{F^{-1}(1)} \bar{F}(s) d s,
$$

then the transform

$\phi_{F}(t)=\frac{1}{\mu} \int_{0}^{F^{-1}(t)} \bar{F}(s) d s .0 \leq t \leq 1$.

is scale invariant and is named the scaled TTT-transform. The scaled TTT-transform and an empirical counterpart, named the TTT-plot, were presented by Barlow and Campo [5] as a tool for identification of failure distribution models. Scaled TTT-transforms for some families of life distributions are given by Barlow and Campo [5], Barlow [30], Bergman and Klefsjo [9], here we present the following theorem to find the scaled TTT-transforms for UBAC(2) class of life 
distributions.

Definition (2.2): The function

$$
\phi_{F}(t)=\frac{H_{F}^{-1}(t)}{H_{F}^{-1}(1)}
$$

is called the scaled TTT-transform. if $F$ is the exponential distribution then TTT-transform is given by

$\phi_{F}(t)=\frac{\int_{0}^{F^{-1}(t)} e^{-u} d u}{\int_{0}^{F^{-1}(1)} e^{-u} d u}=t, 0 \leq t \leq 1$.

Theorem 2.1. Let $F$ and $\phi_{F}(t)$ be a distribution function and scaled TTT-transform, then $F$ is $\operatorname{UBAC}(2)$ if

$$
\begin{gathered}
\frac{1}{\mu} \int_{0}^{F^{-1}\left(w_{1}\right)+F^{-1}\left(w_{2}\right)} \bar{F}(s) d s-\phi_{F}\left(w_{2}\right) \\
\geq \frac{1}{\mu}\left[1-w_{2}\right]\left(1-e^{-F^{-1}\left(w_{1}\right)}\right)
\end{gathered}
$$

Proof: Since $F$ is UBAC(2), then

$$
\begin{gathered}
v(t)-v(x+t) \geq\left(1-e^{-x}\right) \bar{F}(t), \\
\int_{t}^{\infty} \bar{F}(s) d s-\int_{x+t}^{\infty} \bar{F}(s) d s \geq\left(1-e^{-x}\right) \bar{F}(t), \\
\int_{0}^{x+t} \bar{F}(s) d s-\int_{0}^{t} \bar{F}(s) d s \geq\left(1-e^{-x}\right) \bar{F}(t),
\end{gathered}
$$

Let $x=F^{-1}\left(w_{1}\right), t=F^{-1}\left(w_{2}\right), 0 \leq w_{1}, w_{2} \leq 1$.

Then we can write (2.8) as

$$
\begin{aligned}
& \int_{0}^{F^{-1}\left(w_{1}\right)+F^{-1}\left(w_{2}\right)} \bar{F}(s) d s-\int_{0}^{F^{-1}\left(w_{2}\right)} \bar{F}(s) d s \\
& \geq\left[1-w_{2}\right]\left(1-e^{-F^{-1}\left(w_{1}\right)}\right)
\end{aligned}
$$

After dividing (2.9) by $\mu$, hence the theorem is proved.

\section{TEST STATISTIC BASED ON THE SCALED TTT- TRANSFORM}

In this section we present a test statistic using the scaled TTTtransform for testing $H_{0}: \bar{F}$ is exponential against $H_{1}: \bar{F}$ is UBAC(2) and isn't exponential is studied for a random sample $X_{1}, X_{2}, \ldots \ldots, X_{n}$ from a population with distribution function $F$.

We proposed the following measure of departure

$$
\delta_{U_{T}}=\frac{1}{\mu} \int_{0}^{F^{-1}\left(w_{1}\right)+F^{-1}\left(w_{2}\right)} \bar{F}(s) d s
$$

$-\phi_{F}\left(w_{2}\right)-\frac{1}{\mu}\left[1-w_{2}\right]\left(1-e^{-F^{-1}\left(w_{1}\right)}\right)$

Note that under $H_{0}: \delta_{U_{T}}=0$, while under $H_{1}: \delta_{U_{T}} \geq 0$.

Let $X_{(1)} \leq \ldots \leq X_{(n)}$ be the ordered statistics of the independent random sample $X_{1}, \ldots, X_{n}$ and $\bar{X}$ be the sample mean where $X_{(0)}=0$. Then the empirical distribution of the distribution function $F$ is $\hat{F}(x)=F_{n}(x)=\frac{1}{n}\left(X_{(i)} \leq x\right), i=$ $1,2 \ldots, n, \bar{F}(x)$ the survival function is estimated by $\overline{F_{n}}(x)=$ $1-F_{n}(x), H_{F}^{-1}(x)$ the TTT- transform is estimated by $H_{F_{n}}^{-1}(x)=\frac{s_{j}}{n}$, where $S_{j}=\sum_{i=1}^{j} D_{i}, D_{i}=(n-i+1)\left(X_{(i)}\right.$ $\left.X_{(i-1)}\right)$ "the normalized sample spacing" and $\phi_{F}(x)$ the scaled TTT- transform is estimated by $\phi_{F_{n}}(x)=\frac{s_{j}}{n \bar{X}}$. Hence, the estimate of $\delta_{U_{T}}$ is proposed by

$$
\begin{aligned}
\hat{\delta}_{U_{T}}=\frac{1}{n \bar{x}} \sum_{m=1}^{L}(n- & m+1)\left[X_{(m)}-X_{(m-1)}\right] \\
& -\frac{1}{n \bar{x}} \sum_{j=1}^{n}(n-j+1)\left[X_{(j)}-X_{(j-1)}\right] \\
& -\frac{1}{\bar{x}}\left[1-\frac{j-1}{n}\right]\left[1-e^{-X_{(i)}}\right] .
\end{aligned}
$$

where $i=1,2, \ldots, n, j=1,2, \ldots, n$ and $X_{(1)} \leq \ldots \leq X_{(n)}$ are the ordered statistics of the independent random sample $X_{1}, \ldots, X_{n}$.

Note that $\mathrm{L}=\mathrm{i}+\mathrm{j}$ if $i+j<n$ but $\mathrm{L}=\mathrm{n}$ if $i+j \geq n$

To reduce the size of the test statistic we use:

$$
\widehat{\Delta}_{U_{T}}^{*}=\frac{\widehat{\delta}_{U_{T}}}{n^{2}}
$$

\section{MONTE CARLO NULL DISTRIBUTION CRITICAL POINTS}

We calculate, via Monte Carlo method, the empirical critical points of $\hat{\delta}^{*}{ }_{U_{T}}$ in Eq. (3.2) for samples 10(5)100.

Tables (4.1) gives the lower and the upper percentile points for $1 \%, 5 \%, 10 \%, 90 \%, 95 \%, 99 \%$ The calculations are based on 10000 simulated samples of sizes $n=10(5) 100$.

Table (4.1) Critical Values of $\widehat{\Delta}_{U_{T}}^{*}$

\begin{tabular}{|c|c|c|c|c|c|c|}
\hline$n$ & $1 \%$ & $5 \%$ & $10 \%$ & $90 \%$ & $95 \%$ & $99 \%$ \\
\hline 10 & -5.29544 & -5.23303 & -5.19978 & -4.96655 & -4.9333 & -4.87089 \\
\hline 15 & -3.08288 & -3.03193 & -3.00478 & -2.81435 & -2.7872 & -2.73624 \\
\hline 20 & -1.96965 & -1.92552 & -1.90201 & -1.73709 & -1.71358 & -1.66945 \\
\hline 25 & -1.36901 & -1.32954 & -1.30851 & -1.161 & -1.13997 & -1.1005 \\
\hline 30 & -1.01254 & -0.976512 & -0.957313 & -0.822659 & -0.803461 & -0.76743 \\
\hline 35 & -0.784398 & -0.75104 & -0.733265 & -0.6086 & -0.590826 & -0.557468 \\
\hline 40 & -0.629619 & -0.598416 & -0.581789 & -0.465175 & -0.448549 & -0.417346 \\
\hline 45 & -0.519704 & -0.490285 & -0.47461 & -0.364665 & -0.34899 & -0.319571 \\
\hline 50 & -0.43874 & -0.410831 & -0.39596 & -0.291658 & -0.276786 & -0.248877 \\
\hline 55 & -0.377292 & -0.350681 & -0.336502 & -0.237054 & -0.222875 & -0.196265 \\
\hline 60 & -0.329481 & -0.304004 & -0.290428 & -0.195214 & -0.181638 & -0.156161 \\
\hline 65 & -0.291493 & -0.267015 & -0.253972 & -0.162493 & -0.14945 & -0.124972 \\
\hline 70 & -0.260764 & -0.237177 & -0.224608 & -0.136456 & -0.123888 & -0.100301 \\
\hline 75 & -0.235518 & -0.21273 & -0.200588 & -0.115426 & -0.103283 & 0.0804958 \\
\hline
\end{tabular}




\begin{tabular}{|c|c|c|c|c|c|c|}
\hline 80 & -0.214495 & -0.192431 & -0.180674 & -0.0982158 & -0.086459 & -0.064395 \\
\hline 85 & -0.196778 & -0.175373 & -0.163967 & -0.0839708 & -0.0725651 & -0.0511598 \\
\hline 90 & -0.181689 & -0.160887 & -0.149803 & -0.0720603 & -0.0609759 & -0.0401737 \\
\hline 95 & -0.168717 & -0.148469 & -0.137681 & -0.0620118 & -0.051223 & -0.0309757 \\
\hline 100 & -0.157469 & -0.137734 & -0.127219 & -0.0534658 & -0.0429503 & -0.0232156 \\
\hline
\end{tabular}

It is shown from the table that the values of the percentiles decreases when the sample size increases.

Finally, the power of the test statistics $\widehat{\Delta}_{U_{T}}$ is considered for 95\% percentiles in Table (4.2) for three of the most commonly used alternatives [see Hollander and Proschan [31]], they are

(i)Linearfailurerate: $\bar{F}_{\theta}=e^{-x-\frac{\theta x^{2}}{2}}, x>0, \theta>0$

(ii)Makeham: $\bar{F}_{\theta}=e^{-x-\theta\left(x+e^{-x}-1\right)}, x \geq 0, \theta>0$

(iii)Weibull: $\bar{F}_{\theta}=e^{-x^{\theta}}, x \geq 0, \theta>0$

These distributions are reduced to exponential distribution for appropriate values of $\theta$

Power estimates increase when the parameter $\theta$ is far from exponentiality and when the size of the sample $\mathrm{n}$ increases.

\section{APPLYING THE TEST}

\section{Example 1}

Consider real data representing 40 patients suffering from

blood cancer.We use the data as given in Abu-Youssef [32].The ordered life times (in day)are:

$115,181,255,418,441,461,516,739,743,789,807,865$, 924, 983, 1024, 1062, 1063, 1169, 1191, 1222, 1222, 1251, $1277,1290,1357,1369,1408,1455,1478,1549,1578,1578$, 1599, 1603, 1604, 1696, 1735, 1799, 1815, 1852. Using equation (3.2), the value of test statistics, based on the above data is $\widehat{\Delta}_{M_{T}}=-0.870536$. the critical value at $\alpha=0.05$ is -0.448549 This value leads to the rejection of $H_{0}$ at thesignificance level $\alpha=0.05$. Therefore the data has UBAC(2) Property.

\section{Example 2}

Table (4.2) Power Estimate of $\widehat{\Delta}_{U_{T}}$

\begin{tabular}{|c|c|c|c|c|}
\hline \multirow{2}{*}{ Distribution } & $\theta$ & \multicolumn{3}{|c|}{ Sample Size } \\
\cline { 2 - 5 } & & $n=10$ & $n=20$ & $n=30$ \\
\hline \multirow{3}{*}{$\begin{array}{c}\text { Linear failure } \\
\text { rate }\end{array}$} & 2 & 0.99974 & 1 & 1 \\
\cline { 2 - 5 } & 3 & 0.99896 & 1 & 1 \\
\cline { 2 - 5 } & 4 & 0.99702 & 1 & 1 \\
\hline \multirow{3}{*}{$F_{2}$ Makham } & 2 & 0.99978 & 1 & 1 \\
\cline { 2 - 5 } & 3 & 0.99942 & 1 & 1 \\
\cline { 2 - 5 } & 4 & 0.99846 & 1 & 1 \\
\hline \multirow{3}{*}{$F_{3}$ Weibull } & 2 & 1 & 1 & 1 \\
\cline { 2 - 5 } & 3 & 1 & 1 & 1 \\
\cline { 2 - 5 } & 4 & 1 & 1 & \multicolumn{2}{c}{} \\
\hline
\end{tabular}

In an experiment at Florida state university to study the effect of methyl mercury poisoning on the life lengths of fish goldfish were subjected to various dosages of methyl mercury (Kochar [33]). At one dosage level the ordered times to death in week are:
$6,6.143,7.286,8.714,9.429,9.857,10.143,11.571,11.714$ 11.714

The value of test statistics, based on the above data is $\widehat{\Delta}_{U_{T}}=-0.875165$. the critical value at $\alpha=0.05$ is -4.9333 . Then $H_{0}$ at the significance level $\alpha=0.05$ is rejected. Therefore the data has UBAC(2) Property.

\section{Example 3}

The following data represent 39 liver cancers patients taken from El Minia Cancer Center Ministry of Health Egypt Attia et al [34]. The ordered life times (in days) are:

$107,18,74,20,23,20,23,24,52,105,60,31,75$, $107,71,107,14,49,10,15,30,26,14,87,51,17$, $116,67,20,14,40,14,30,96,20,20,61,150,14$

The value of test statistics, based on the above data is $\widehat{\Delta}_{M_{T}}=-0.930386$. the critical value at $\alpha=0.05$ is -0.472933 , then we reject $H_{0}$ at the significance level $\alpha=0.05$.Therefore the data has UBAC(2) Property.

\section{CONCLUSION}

In this paper, Testing exponentiality versus used better than aged in increasing concave ordering $\operatorname{UBAC}(2)$ based on total time on test (TTT)-Transform is proposed. The percentiles of proposed test are tabulated in table (4.1), which clearly show the critical values of the estimate of departure from measure is decreasing as the sample size increasing. This test is more efficient than the test for (UBAC). It gives a very good power for most alternatives.

\section{FUNDING}

The authors declare that they had no funding.

\section{AUTHORS' CONTRIBUTIONS}

The authors read and approved the final manuscript.

\section{COMPETING INTERESTS}

The authors declare that they have no competing interests.

\section{ACKNOWLEDGEMENTS}

The authors would like to thank the editors and the referees for their valuable comments and suggestions.

\section{REFERENCES}

[1] Bryson, M. C. and Siddiqui, M. M. 1969. Some criteria for aging. Journal of the American Statistical Association, Vol. 64, No. 328, pp. 1472-1483.

[2] Barlow, R. E. and Proschan, F. 1981. Mathematical theory of reliability. To Begin With: Silver-Spring, MD.

[3] Abu-Youssef, S. E. 2004. Non-parametric Test for Monotone Variance Residual Life Class of Life Distributions with Hypothesis Testing Applications. Applied Mathematics and Computations, Vol. 158, No. 3, pp. 817-826.

[4] Ahmed, I. A. 2004. Some Properties of Classes of Life Distributions with Unknown Age. Statistics and Probability Letters, Vol. 69, No. 3, pp. 333-342. 
[5] Barlow, R. E. and Campo, R. 1975. Total Time on Test Processes and Application to Failure Data Analysis. Reliab. Fault Tree Analysis, SIAM, Philadephia, Vol. 8, No. 4, pp. 451-481.

[6] Klefsjo, B. 1980. Some Aging Properties and The Total Time on Test Transform. Res. Rep., Dept. of Math. Statist. Univ. of Umea, Sweden.

[7] Klefsjo, B. 1982. The HNBUE and HNWUE classes of life distributions. Naval Logist Res. Quart, Vol. 29, pp. 331-344.

[8] Klefsjo, B. 1983. Some Tests Against Aging Based on Total Time on Test Transform. Comm. Statist., Theor. Meth., Vol.12, No. 8, pp. 907-927.

[9] Bergman, B. and Klefsjo, B. 1984. The total time on test concept and its use in reliability theory. Operat. Res., Vol. 32, No. 3, pp. 596-606.

[10] Pham, T. G. and Turkkan, M. 1994. The Lorenz and the scaled total-time-on-test transform curves: a unified approach. IEEE Trans. Reliab., Vol. 43, No. 1, PP. 7684.

[11] Bartoszewicz, J. 1995. Stochastic order relations and the total time on test transform. Ŝtatist. Prob. Lett., Vol. 22, No. 2, pp. 103-110.

[12] Bartoszewicz, J. 1996. Tail orderings and the total time on test transform. Appl. Math., Vol, 24, No. 1,pp. $77-$ 86.

[13] Haupt, E. and Schabe, H. 1997. The TTT transformation and a new bathtab distribution model. J. Statist. Planning Infer., Vol. 60, No. 2, pp. 229-240.

[14] Kochar, S. C., Li, X., and Shaked, M. 2002. The total time on test transform and the excess wealth stochastic orders of distributions. Adv. Appl. Prob., Vol. 34, No. 4, pp. 826-845.

[15] Li, X. and Zou, M. 2004. Preservation of stochastic orders for random minima and maxima with applications. Naval Res. Logistics., Vol. 51, No. 3, pp. 332-334.

[16] Ahmed, I. A., Li, X. and Kayid, M. 2005. The NBUT class of life distributions. IEEE Trans. Reliab., Vol. 54, No. 3, pp. 396-401.

[17] Li, H. and Shaked, M. 2007. A general family of univariate stochastic orders. J. Statist. Planning Infer., Vol. 137, No. 11, pp. 3601-3610.

[18] Al-Nachawati, H. 2007. Test for Monotone Variance Residual Life Class of Life Distributions Based on Total Time on Test Transformation. J. King Saud Univ., Vol. 19, No. 2, pp. 109-117.

[19] Nanda, A. K. and Shaked, M. 2008. Partial ordering and aging properties of order statistics when sample size is random: a brief review. Commun. Statist. Theory Meth., Vol. 37, No. 11, pp. 1710-1720.

[20] Abu-Youssef, S. E., Mohie El-Din, M. M. and Hassan, M. KH. 2012. Testing Of EBELC Classes Of Life Distributions Based On TTT - Transform. International
Journal of Reliability and Applications., Vol. 13, No. 1, pp. 49-56.

[21] Mohie El-Din, M. M., Abu-Youssef, S. E. and 2013. Testing unknown age classes of life distributions based on TTT-transform. International Journal of Reliability and Applications., Vol. 14, No. 1, pp. 1-9.

[22] Ali, N. S. A. 2018. On the Properties of the UBAC(2) Class of Life Distributions. Journal of Testing and Evaluation., Vol. 46, No. 2, pp.730-735.

[23] Willmot, G. and Cai, J. 2000. On classes of life time distributions with unknown age. Probability in the engineering and informational sciences, Vol. 14, No. 4, pp. 473-484.

[24] Dicrescenzo, A. 1999. Dual stochastic ordering, decreasing aging properties of divices of unknown age. it Communications in Statistics. Stochastic Models, Vol 15 , No. 3 , pp. $561-576$.

[25] Mohie El-Din, M. M., Abu-Youssef, S. E. and Ali, N. S A. 2015. A New Class of Life Distributions Based On Unknown Age, IJRA, Vol. 16, No. 1, pp. 27-34.

[26] Deshpand, J. V., Kocher, S. C. and Singh, H. 1986. Aspects of Positive Aging. J. Appl. Probab., Vol. 28, No. 3, pp. 1472-1483.

[27] Deshpand, J. V. and Purohit, S. G. 2005. Life Time: Statistical Models and Methods, World Scientific Publishing Co., Singapore. Vol. 11.

[28] Barlow, R. E. and Doksum, K. 1972. Isotonic tests for convex ordering. In Proceedings of the Sixth Berkeley Symposium on Mathematical Statistics and Prob. Univ. of Calif., Vol. 1, pp. 293-323.

[29] Barlow, R. E., Bartholomew, D. J., Bremner, J. M. and Brunk, H. D. 1972. Statistical inference under order restriction. Wiley, New York, Vol. 27, No. 4, pp. 139189.

[30] Barlow, R. E. 1979. Geometry of the total time on test transform, Naval Res. Logist. Quart., Vol. 26, No. 3, pp. 393-402.

[31] Hollander, M. and Prochan, F. 1975. Test for mean residual life. Biometrika. 62(3), pp. 585-593.

[32] Abu-Youssef, S. E. 2009. A Goodness of Fit Approach to Monotone Variance Residual LifeClass of Life Distributions, Applied Mathematical Sciences., Vol. 3, no. $15,715-724$

[33] Kochar, S. C. 1985. Testing exponentiality against monotone failure rate average. Comm. in Stat. - Theo and Meth., Vol. 14, No. 2, pp. 381-392.

[34] Attia, A. F., Mahmoud, M. A. W. and Abdul-Moniem, I. B. 2004. On Testing for Exponential Better than Used in Average Class of Life Distributions Based on the UTest. The proceeding of The 39 th Annual Conference on Statistics. Computer Sciences and Operation Research, ISSR Cairo University-Egypt, pp. 11-14. 